\title{
DIFFERENTIAL OPERATORS WITH NONSINGULAR CHARACTERISTICS
}

\author{
BY LARS HÖRMANDER ${ }^{1}$ \\ Communicated by R. P. Boas, November 25, 1961
}

1. Introduction. This note is devoted to proof and application of estimates of the form

$$
\begin{aligned}
& \sum_{|\alpha|<m} \tau \int\left|D^{\alpha} u\right|^{2} e^{2 \tau \phi} d x \leqq K_{1} \int|P(x, D) u|^{2} e^{2 \tau \phi} d x \\
& +K_{2} \sum_{|\alpha|<m-1} \tau^{2(m-|\alpha|)-1} \int\left|D^{\alpha} u\right|^{2} e^{2 \tau \phi} d x, \quad u \in C_{0}^{\infty}(\Omega), \tau>\tau_{0},
\end{aligned}
$$

where $P$ is a linear differential operator of order $m$ in an open set $\Omega$ in $R_{n}$ and $\phi$ is a fixed function. (For all other notations we refer to Hörmander [4].) From the estimate (1.1) with $K_{2}=0$ we obtain theorems on uniqueness for the Cauchy problem for the operator $P(x, D)$ by means of a classical method of Carleman [2]. These extend the results of Calderón [1]. Using the estimate (1.1) with an arbitrary constant $K_{2}$ we can prove existence theorems for the differential equation $P(x, D) u=f$ which improve those of Hörmander [3]. The estimate (1.1) also implies results on unique continuation of singularities of solutions of the differential equation $P(x, D) u=0$ such as have been given by John [6] and Malgrange [8] for operators with constant coefficients. For the importance of such results in connection with existence theorems for the differential equation $P(x, D) u=f$ when $f$ is an arbitrary distribution, as well as for all details of proof, we refer the reader to Hörmander [5].

2. The a priori estimate (1.1). Let all coefficients of $P$ be in $L^{\infty}$ and those of the principal part $P_{m}$ be in $C^{1}$. Also assume that $\phi$ is in $C^{2}$ and write

$$
P_{m}^{(j)}(x, \xi)=\partial P_{m}(x, \xi) / \partial \xi_{j} ; \quad P_{m, j}(x, \xi)=\partial P_{m}(x, \xi) / \partial x_{j} .
$$

Theorem 2.1. Let $N=\operatorname{grad} \phi(x)$ where $x \in \Omega$ and let $\zeta=\xi+i \sigma N$, with $\xi \in R_{n}$ and $0 \neq \sigma \in R_{1}$, satisfy the characteristic equation

$$
P_{m}(x, \zeta)=0 .
$$

If (1.1) is valid, it then follows that

${ }^{1}$ This work was supported by NSF research grants G-14362 and G-10093 at Madison, Wisconsin. A summary of the results was given at the AMS conference on Functional Analysis at Stanford in August 1961. 


$$
|\zeta|^{2(m-1)}-K_{2} \sigma^{2}\left(|\zeta|^{2}+\sigma^{2}\right)^{m-2} \leqq 2 K_{1}\left\{\sum_{j, k=1}^{n} \frac{\partial^{2} \phi}{\partial x_{j} \partial x_{k}} P_{m}^{(j)}(x, \zeta) \overline{P_{m}^{(k)}(x, \zeta)}\right.
$$

$$
+(2 i \sigma)^{-1} \sum_{1}^{n}\left(P_{m, k}(x, \zeta) \overline{P_{m}^{(k)}(x, \zeta)}-P_{m}^{(k)}(x, \zeta) \overline{\left.P_{m, k}(x, \zeta)\right)}\right\},
$$

when the left-hand side is positive.

This follows by applying (1.1) to functions of the form

$$
u(x)=\exp (i \tau w(x) / \sigma) \psi\left(x \tau^{1 / 2}\right)
$$

where $w(x)=\langle x, \zeta\rangle+O\left(|x|^{2}\right)$ when $x \rightarrow 0$. When $\tau \rightarrow \infty$ a differential inequality for $\psi$ is obtained which leads to (2.2). (A similar but less precise discussion occurs in Hörmander [4, pp. 216-217].)

Note that (2.2) is applicable for every $\zeta$ satisfying (2.1) if $K_{2}=0$, but only for nearly real $\zeta$ if $K_{2}$ is large. An elementary algebraic discussion shows that Theorem 2.1 implies

CoROLlaRy 2.1. Let the assumptions of Theorem 2.1 be fulfilled and assume that $P_{m}$ has real coefficients. If $x \in \Omega$ and $0 \neq \xi \in R_{n}$ is a solution of the equations

$$
P_{m}(x, \xi)=\sum_{1}^{n} P_{m}^{(j)}(x, \xi) \partial \phi / \partial x_{j}=0
$$

but $P_{m}^{(j)}(x, \xi) \neq 0$ for some $j$, it follows that

$$
\begin{aligned}
|\xi|^{2(m-1)} & \leqq 2 K_{1}\left\{\sum_{j, k=1}^{n} \partial^{2} \phi / \partial x_{j} \partial x_{k} P_{m}^{(j)}(x, \xi) P_{m}^{(k)}(x, \xi)\right. \\
+ & \left.\sum_{j, k=1}^{n}\left(P_{m, j}^{(k)}(x, \xi) P_{m}^{(j)}(x, \xi)-P_{m, j}(x, \xi) P_{m}^{(j k)}(x, \xi)\right) \partial \phi / \partial x_{k}\right\} .
\end{aligned}
$$

The geometric significance of the positivity of the right-hand side of (2.4) is that the restriction of $\phi$ to any real bicharacteristic curve has a positive second derivative where the first derivative vanishes.

The conditions (2.2) and (2.4) are nearly sufficient for the validity of (1.1). For simplicity in statements we limit ourselves here to the case when $P_{m}$ has real coefficients; if $P_{m}$ does not have real coefficients and is not elliptic, we need the same additional hypothesis as in Theorem 4.3 of Hörmander [3].

THEOREM 2.2. Let $\Omega$ be a bounded open set, $\phi$ a real valued function in $C^{\infty}(\bar{\Omega})$ with $\operatorname{grad} \phi(x) \neq 0$ when $x \in \bar{\Omega}$, and $P(x, D)$ a differential 
operator of order $m$ with bounded measurable coefficients such that the principal part $P_{m}(x, D)$ has real coefficients belonging to $C^{1}(\bar{\Omega})$. A ssume further that

$$
\begin{aligned}
& \sum_{j, k=1}^{n} \partial^{2} \phi / \partial x_{j} \partial x_{k} P_{m}^{(j)}(x, \xi) P_{m}^{(k)}(x, \xi) \\
& \quad+\sum_{j, k=1}^{n}\left(P_{m, j}^{(k)}(x, \xi) P_{m}^{(j)}(x, \xi)-P_{m, j}(x, \xi) P_{m}^{(j k)}(x, \xi)\right) \partial \phi / \partial x_{k}>0
\end{aligned}
$$

if $x \in \bar{\Omega}$ and $0 \neq \xi \in R_{n}$ satisfy the characteristic equation $P_{m}(x, \xi)=0$ and

$$
\sum_{1}^{n} P_{m}^{(j)}(x, \xi) \partial \phi / \partial x_{j}=0
$$

Then there is a constant $K$ such that when $\tau$ is sufficiently large

$$
\begin{aligned}
& \sum_{|\alpha|<m} \tau^{2(m-|\alpha|)-1} \int\left|D^{\alpha} u\right|^{2} e^{2 \tau \phi} d x \\
& \quad \leqq K \int\left\{|P(x, D) u|^{2}+\tau^{2 m-1}|u|^{2}\right\} e^{2 \tau \phi} d x, \quad u \in C_{0}^{\infty}(\Omega) .
\end{aligned}
$$

TheOREM 2.3. Assume that, in addition to the hypotheses of Theorem 2.2, we have

$$
\sum_{j, k=1}^{n} \partial^{2} \phi / \partial x_{j} \partial x_{k} P_{m}^{(j)}(x, \zeta) \overline{P_{m}^{(k)}(x, \zeta)}
$$

$$
+(2 i \tau)^{-1} \sum_{1}^{n}\left(P_{m, k}(x, \zeta) \overline{P_{m}^{(k)}(x, \zeta)}-P_{m}^{(k)}(x, \zeta) \overline{P_{m, k}(x, \zeta)}\right)>0
$$

if $\zeta=\xi+i \tau$ grad $\phi(x)$, with $x \in \bar{\Omega}, \xi \in R_{n}$ and $0 \neq \tau \in R_{1}$, satisfies the characteristic equation $P_{m}(x, \zeta)=0$. Then there is a constant $K$ such that for sufficiently large $\tau$

$$
\begin{array}{r}
\sum_{|\alpha|<m} \tau^{2(m-|\alpha|)-1} \int\left|D^{\alpha} u\right|^{2} e^{2 \tau \phi} d x \leqq K \int|P(x, D) u|^{2} e^{2 \tau \phi} d x, \\
u \in C_{0}^{\infty}(\Omega) .
\end{array}
$$

It is sufficient to prove these theorems locally assuming that $\phi$ is a linear function, $\phi(x)=\langle x, N\rangle$ with a constant $N$. We then start with the trivial estimate, where $v(x)=u(x) e^{\tau\langle x, N\rangle}$, 


$$
\begin{aligned}
\int\left|P_{m}(x, D) u\right|^{2} e^{2 \tau\langle x, N\rangle} d x=\int\left|P_{m}(x, D+i \tau N) v\right|^{2} d x \\
\geqq \int\left\{\left|P_{m}(x, D+i \tau N) v\right|^{2}-\left|P_{m}(x, D-i \tau N) v\right|^{2}\right\} d x .
\end{aligned}
$$

In the right-hand side it is possible to make an integration by parts so that only derivatives of $v$ of order $\leqq m-1$ occur afterwards. The error committed in fixing the argument in the coefficients of this form can then be estimated by means of derivatives of $v$ of order $\leqq m-1$ and the estimate (1.1) can thus be obtained by means of Fourier transforms in a way similar to the standard estimates for elliptic operators.

3. Uniqueness of the Cauchy problem. From Theorem 2.3 and the usual argument of Carleman [2] we obtain

Theorem 3.1. Let $P(x, D)$ be a differential operator of order $m$ with bounded measurable coefficients in some open neighbourhood $\Omega$ of a point $x^{0}$, and assume that the coefficients of $P_{m}$ are in $C^{1}$ and are real. Let $\phi$ be a real-valued function in $C^{2}$ such that $N^{0}=\operatorname{grad} \phi\left(x^{0}\right) \neq 0$ and

$$
\begin{aligned}
& \sum_{j, k=1}^{n} \partial^{2} \phi / \partial x_{j} \partial x_{k} P_{m}^{(j)}(x, \zeta) \overline{P_{m}^{(k)}(x, \zeta)} \\
& \quad+(2 i \tau)^{-1} \sum_{1}^{n}\left(P_{m, k}(x, \zeta) \overline{P_{m}^{(k)}(x, \zeta)}-P_{m}^{(k)}(x, \zeta) \overline{\left.P_{m, k}(x, \zeta)\right)}>0\right.
\end{aligned}
$$

if $x=x^{0}$ and $0 \neq \xi=\xi+i \tau N^{0}$ with $\xi \in R_{n}$ and $\tau \in R_{1}$ is a solution of

$$
P_{m}\left(x^{0}, \zeta\right)=0, \quad \sum_{1}^{n} P_{m}^{(j)}\left(x^{0}, \zeta\right) N_{j}^{0}=0 .
$$

If $u \in C^{m}(\Omega)$ satisfies the equation $P(x, D) u=0$ and $u=0$ when $\phi(x)$ $>\phi\left(x^{0}\right)$, it then follows that $u=0$ in a neighbourhood of $x^{0}$.

Note that the left hand side of (3.1) is a polynomial in $\xi$ and $\tau$ since the coefficients of $P_{m}$ are real. Hence it makes sense also if $\tau=0$.

The hypothesis of Calderón [1] is that (3.2) has no solution $\zeta \neq 0$. Counterexamples due to $\mathrm{P}$. Cohen show that the convexity assumption (3.1) is vital at least as far as real solutions $\zeta$ of (3.2) are concerned.

4. Existence theorems. Let $H_{(s)}$ be the space of all distributions $u \in \mathcal{S}^{\prime}\left(R_{n}\right)$ such that

$$
\|u\|_{(s)}^{2}=(2 \pi)^{-n} \int|\mathfrak{u}(\xi)|^{2}\left(1+|\xi|^{2}\right)^{\bullet} d \xi<\infty .
$$


The methods of $\$ 6$ in Hörmander [3] can be used to show that it is possible to pass from the $L^{2}$ norms in (1.1) to $H_{(s)}$ norms for an arbitary real $s$. (The possibility to choose the parameter $\tau$ large is very important here.) We thus obtain the following theorem where the existence statement follows by duality.

Theorem 4.1. Let $P$ be a differential operator of order $m$ with $C^{\infty}$ coefficients and let $\phi$ be a $C^{\infty}$ function such that (1.1) is valid, at least for all $u$ with support in fixed compact subsets of $\Omega$. If $u$ is a distribution with compact support in $\Omega$ such that $P(x, D) u=0$, it then follows that $u \in C_{0}^{\infty}(\Omega)$. The set of such functions $u$ with support in a fixed compact subset of $\Omega$ is finite dimensional. Let $\Omega^{\prime}$ be a relatively compact open subset of $\Omega$ and $f \in H_{(s)}$. If

$$
f(v)=0
$$

for every $v \in C_{0}^{\infty}(\Omega)$ with support in $\bar{\Omega}^{\prime}$ such that $P(x, D) v=0$, it then follows that one can find $u \in H_{(s+m-1)}$ such that ${ }^{t} P(x, D) u=f$ in $\Omega^{\prime}$. Here ${ }^{t} P$ is the adjoint of $P$.

Thus we have a local existence theorem for every $P$ satisfying the assumptions of Theorem 2.2 for some $\phi$. In view of the methods of Malgrange [7], Theorems 3.1 and 4.1 together give global existence theorems for the differential equation ${ }^{t} P(x, D) u=f$ if $f$ is an arbitrary distribution of finite order and the boundary of $\Omega$ satisfies the condition in Theorem 3.1.

5. Unique continuation of singularities. From the estimate (1.1) we can also obtain the following result.

Theorem 5.1. Let $P$ be a differential operator of order $m$ with $C^{\infty}$ coefficients and real coefficients in the principal part, defined in an open neighbourhood $\Omega$ of a point $x^{0}$. Let $\phi$ be a function in $C^{2}(\Omega)$ such that $\operatorname{grad} \phi\left(x^{0}\right) \neq 0$ and $(2.5)$ is valid when $x=x^{0}$ for every real $\xi \neq 0$ satisf $y$ ing the characteristic equation $P_{m}(x, \xi)=0$ and (2.6). If $u \in D^{\prime}(\Omega)$ and

$$
\begin{aligned}
u \in C^{\infty}\left(\Omega^{+}\right) \text {where } \Omega^{+} & =\left\{x ; x \in \Omega, \phi(x)>\phi\left(x^{0}\right)\right\} \\
P(x, D) u & =f \in C^{\infty}(\Omega),
\end{aligned}
$$

it then follows that $u \in C^{\infty}\left(\Omega^{\prime}\right)$ in a neighbourhood $\Omega^{\prime}$ of $x^{0}$.

If $P$ has constant coefficients and $\phi$ is strictly convex, this result is due to Malgrange [8] and John [6], but for other functions $\phi$ the result is new even for the wave equation. That the convexity of $\phi$ along bicharacteristics which is assumed in Theorem 5.1 is essential follows from an example of Zerner [9] (which can also easily be extended). 
The fairly technical proof of Theorem 5.1 proceeds as follows. We may assume that $\phi(x)=x_{n}$. Using the methods of $\S \S 5-6$ in Hörmander [3] we can then replace the $L^{2}$ norms in (1.1) by norms of the kind

$$
\int\left\|u\left(x_{n}\right)\right\|_{\left(a x_{n}+b\right)}^{2} d x_{n}
$$

where $a$ and $b$ are constants and $\left\|u\left(x_{n}\right)\right\|_{(s)}$ is the norm (4.1) of the function $u$ of $x_{1} \cdots x_{n-1}$ when $x_{n}$ is fixed. If we apply these estimates to the function $u$ in Theorem 5.1 , after multiplication by a function in $C_{0}^{\infty}(\Omega)$, the desired result follows.

\section{REFERENCES}

1. A. P. Calderon, Uniqueness in the Cauchy problem for partial differential equations, Amer. J. Math. 80 (1958), 16-36.

2. T. Carleman, Sur un probleme d'unicité pour les systèmes d'équations aux dérivées partielles d̀ deux variables indépendantes, Ark. Mat. Astr. Fys. 26B (1939), no. 17, 1-9.

3. L. Hörmander, Differential operators of principal type, Math. Ann. 140 (1960), 124-146.

4. - On the uniqueness of the Cauchy problem. I, Math. Scand. 6 (1958), 213-225.

5. - Linear differential operators, Monograph, to appear.

6. F. John, Continuous dependence on data for solutions of partial differential equations with a prescribed bound, Comm. Pure Appl. Math. 13 (1960), 551-585.

7. B. Malgrange, Existence et approximation des solutions des équations aux dérivées partielles et des équations de convolution, Ann. Inst. Fourier Grenoble 6 (1955-1956), 271-355.

8. - Sur la propagation de la régularité des solutions des équations d̀ coefficients constants, Bull. Math. Soc. Sci. Math. Phys. R. P. Roumaine, to appear.

9. M. Zerner, Solutions de l'équation des ondes présentant des singularités sur une droite, C. R. Acad. Sci. Paris 250 (1960), 2980-2982.

UnIVERSITY OF STOCKHOLM 